\title{
Transcriptional Abundance of Myosin Light Chain 2 Gene in Cardiac Differentiated Canine Induced Pluripotent Stem Cells
}

\author{
Tanmay Mondal ${ }^{1}$, Purnima Singh ${ }^{1}$, Pranay Kumar Konda ${ }^{1}$, Kuldeep Kumar ${ }^{1}$, Swati Dubey ${ }^{1}$, \\ Mokshata Gupta ${ }^{2}$, Mihir Sarkar ${ }^{1}$ and Sadhan Bag ${ }^{1^{*}}$ \\ ${ }^{1}$ Division of Physiology and Climatology, Indian Veterinary Research Institute, Izzatnagar, Bareilly, INDIA \\ ${ }^{2}$ Division of Animal Nutrition, Indian Veterinary Research Institute, Izzatnagar, Bareilly, INDIA \\ *Corresponding author: S Bag; E-mail:rplab1ivri@gmail.com
}

Received: 16 Jan., 2020

Revised: 16 Feb., 2020

Accepted: 18 Feb., 2020

\begin{abstract}
Induced pluripotent stem cells (iPSCs) are promising cell source for cardiac tissue engineering and cell based therapies for heart repair as they can be expanded in vitro and differentiated into most cardiovascular cell types, including cardiomyocytes. During embryonic heart development, this differentiation occurs under the influence of internal and external stimuli that guide cells to go down the cardiac lineage. The aim of this study was to characterize the cardiac differentiation potential of a canine iPS cell. With the use of a standard embryoid body-based differentiation protocol for iPS cells were differentiated for 24 days. In vitro differentiations of canine iPSCs via embryoid bodies (EBs) were produced by 'Hanging Drop' method. EB's were differentiated using 5-azacytidine (5-Aza). During differentiation, EBs were collected on day 4, 6, 8, 12, 16, 20 and 24 to evaluate the expression of cardiomyocyte specific marker. Analyses on molecular, structural, and functional levels demonstrated that iPS cell- derived cardiomyocytes show typical features of ES cell- derived cardiomyocytes. Reverse transcription polymerase chain reaction analyses demonstrated expression of marker genes. The differentiated cells expressed cardiac-specific gene myosin light chain 2 (MYL2) which started from day 8 of differentiation and highest expression was observed on day 16. Immunocytochemistry and relative expression of cardiac specific genes revealed that iPS cells differentiate into functional cardiomyocytes and allow to derivation of autologous functional cardiomyocytes for cellular cardiomyoplasty and myocardial tissue engineering.
\end{abstract}

Keywords: Canine iPSC, Cardiomyocyte, 5-Azacytidine, MYL2, EBs

Heart failure is the foremost cause of death in most industrialized countries and a rising cause of death in emerging countries (Moran et al., 1980). The common cause of heart failure is myocardial infraction (MI), which is a damage of myocardium because of oxygen and nutrient insufficiency and replacement with non-contractile scar tissue. The primary challenge in developing therapies for cardiac muscle restoration is the limited regenerative capability of the myocardium (Ivan et al., 2015). While the recent research has established that the adult heart contains a subpopulation of multipotent stem cells capable of heart regeneration (Beltrami et al., 2003), only 1\% at the most of the cardiomyocytes are replaced annually and thus cannot repair the significant cardiac damage that occur during MI. Almost 95\% heart diseases in canine are acquired of which $75 \%$ are valvular diseases and dialated cardiomyopathy. Being the most common cause of heart failure in dog's chronic mitral valve disease has the highest occurrence and account for $75-80 \%$ cardiac diseases in dogs (Wess et al., 2017). Cardiac tissue engineering and cell based therapies, including direct cell injection, are potencial approaches for treating cardiac disease.

The breakthrough report of Takahashi et al. (2007) on generation of iPSCs has revolutionized the field of medicine by introducing alternative to the embryonic stem cells (ESCs). The differentiation of iPSCs into specific cell types

How to cite this article: Mondal, T., Singh, P., Konda, P.K., Kumar, K., Dubey, S., Gupta, M., Sarkar, M. and Bag, S. (2020). Transcriptional abundance of myosin light chain 2 gene in cardiac differentiated canine induced pluripotent stem cells. J. Anim. Res., 10(2): 173-179. 
has great potential for clinical applications regenerative medicine, in-vitro disease modeling, and drug screening (Karakik es et al., 2015). Canine cardiomyocytes derived from pluripotent stem cells are the only viable source for new cardiomyocytes currently available. Presently, though the pharmaceutical and surgical treatment interventions have made progress in the treatment of heart diseases, the prognosis of heart failure remains very poor. The swift evolution of stem cells as a therapeutic alternative has permitted replacement of myocardial cells with cardiomyocytes that are differentiated from stem cells for treating advanced stage of heart failure (Murry et al., 2006). Patient-specific cardiomyocytes can easily be obtained by adult somatic cell reprogramming to generate iPSCs and their subsequent differentiation into cardiomyocytes. Direct injection of iPSCs into the heart presents a drawback as there is a possibility of teratoma formation. However, the scarce ability of iPSCs to differentiate into myocardial cells in vitro necessitates an effective cardiac induction method with reproducibility. Currently available ciPSC-derived cardiomyocytes differentiation protocols leads to generation of primitive cardiomyocytes (Singh et al., 2019; Shimada et al., 2010). Many of the cardiac sarcomeric proteins acquire their chamberspecific expression patterns during late developmental stages (Lyons, 1994). However, the ventricular myosin light chain-2 isoform (MLC-2v) ventricular specification occurs relatively early during mammalian cardiogenesis (O'Brien et al., 1993). Contrarily, the atrial myosin light chain-2 (MLC-2a) expression occurs in the presumptive ventricle prior to MLC-2v, and subsequently it gets downregulated (Kubalak et al., 1994). During fetal stage, MLC$2 \mathrm{a}$ is mostly found in the atria while MLC-2v expression is restricted to the ventricles, although both MLC-2a and MLC-2v persist in low level in the inflow tract, the atrioventricular canal and the outflow tract (Franco et al., 1999).

Several groups have reported that 5-azacytidine, a demethylating agent, induced the differentiation of mesenchymal stem cells into cardiomyocytes in vitro (Fukuda, 2003; Singh et al., 2019) reported that 5 -azacytidine induced the differentiation of ciPSCs into cardiomyocytes. This compound can cause extensive demethylation of 5-methylcytosine and reduce DNA methyltransferase activity in the cell (Haaf and Schmid, 2000). Recently, 5-azacytidine was reported to reverse the differentiation status of EBs back to ES cells (TsujiTakayama et al., 2004). 5-Azacytidine has been useful for studying the roles of DNA methylation in the mechanisms of gene activation and cell differentiation.

In the current study, 5-azacytidine (5-aza) was explored to induce differentiation of canine induced pluripotent stem cells (ciPSC) into myocardial cells through hanging drop method, to establish an effective and safe cardiomyocyte induction method and channelize the theoretical basis for stem cell replacement therapy in end-stage cardiovascular disease.

\section{MATERIALS AND METHODS}

\section{Canine induced pluripotent stem cell derivation}

All studies used ciPSC colonies derived from canine adipose tissue derived mesenchymal stem cell, which was maintained in the undifferentiated state at reproductive physiology laboratory, physiology and climatology division, IVRI, Izatnagar using embryonic fibroblast conditioned medium. During the experiment the canine induced pluripotent stem (ciPSC) cells, early passage (P-10) were taken from available stock of reproductive physiology laboratory at IVRI.

\section{Culture and cardiomyocytes differentiation from Canine iPSCs}

ciPSCs were cultured with Knock Out DMEM/F12 (GIBCO: 10829-018) supplemented with 20\% fetal calf serum, $1 \%$ non-essential amino acids, $2 \mathrm{mmol} / \mathrm{l}$ L-glutamine, and $100 \mu \mathrm{mol} / \mathrm{l} \beta$-ME, LIF and BFGF. Differentiation of ciPSC was carried out by hanging drop culture using 5-azacytidine (Fig. 1). Briefly, a single cell suspension of ciPSCs $\left(2.5 \times 10^{4}\right.$ cells $\left./ \mathrm{ml}\right)$ was prepared in differentiation media consisting of IMDM supplemented with FBS 10\%, NEAA (100X) 0.5\%, $100 \mu \mathrm{MB}-M e r c a p t o e t h a n o l$ and $0.1 \mu \mathrm{M}$ 5-Azacytidine. From this cell suspension, $30 \mu \mathrm{l}$ was pipetted on to the lid of a tissue culture dish and inverted over its bottom dish containing $5 \mathrm{ml}$ phosphate-buffered saline. On day 4, the embryo bodies (EBs) were transferred on gelatin coated 6 well tissue culture plates and grown up to day 24, with periodical changing of the media. During differentiation, EBs was collected on day 4, 6, 8, 12, 16, 20 and 24 for 
the characterization of cardiomyocytes specific marker expression.

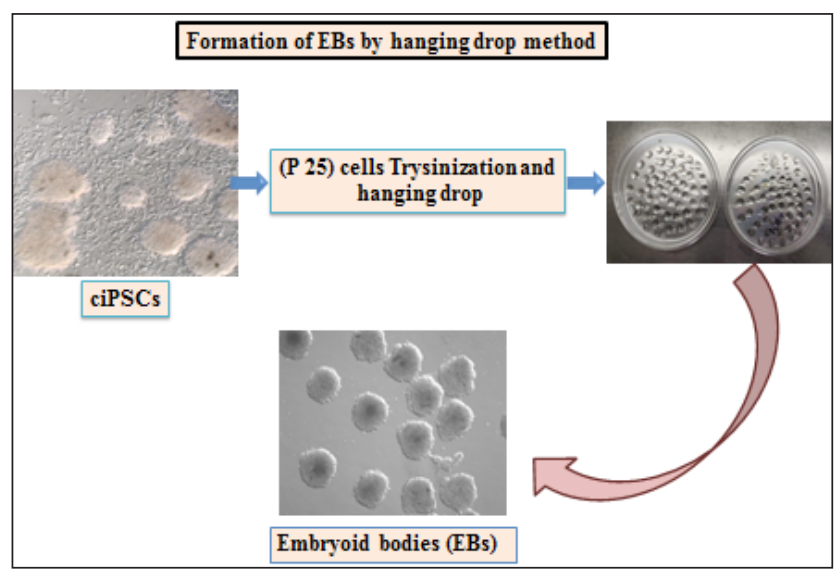

Fig. 1: In-vitro differentiation of canine iPSCs via embryoid bodies produced by 'Hanging Drop' method

\section{Molecular characterization}

The EBs collected at different time points were subjected for total RNA extraction using Trizol method and cDNA was prepared using cDNA Synthesis kit (iScript cDNA Synthesis kit, Bio-Rad). Reverse transcriptase-polymerase chain reaction (RT-PCR) and semi-quantitative gene expression analysis was carried out using Real-Time PCR system (Bio-Rad, USA) with DyNAmo Flash SYBER Green qPCR kit (Thermo Fisher Scientific) and the canine specific primers for MYL 2: F-GGCGCCAA TTCCAACGTGTTCTC, R-GCCCAAGAGCAGCAA AGGTGTC, at annealing temperature of $60{ }^{\circ} \mathrm{C}$. The glyceraldehyde 3-phosphate dehydrogenase (GAPDH) was taken as endogenous housekeeping control gene, for which the primers were F-CCATCTTCCAGGAG CGAGAT, R-TTCTCCATGGTGGTGAAGAC and annealing temperature of $55 \mathrm{C}^{\circ}$. Each PCR product was size-fractionized by $2 \%$ agarose gel electrophoresis and bands were visualized with a UV trans-illuminator (Bio $\mathrm{Rad})$.

\section{Cytochemical staining}

Immunocytochemistry was done as per the methods of National Institutes of Health, resource for stem cell research, USA. For immunocytochemistry, cells were fixed with $4 \%$ paraformaldehyde in PBS and permeabilized with $0.2 \%$ Triton $\mathrm{X}-100$ in PBS (Sigma-Aldrich). The cells were then blocked with $2 \%$ bovine serum albumin in PBS and incubated with goat polyclonal primary antibody viz Connexin43 (Cx43) (Santa Cruz Biology, USA). The cells were washed and then incubated with fluorescencelabeled anti goat secondary antibodies (life technologies, USA; 1:200) and DAPI to stain the nuclei. The negative control was performed following the entire procedurue, except adding primary antibodies.

\section{RESULTS AND DISCUSSION}

In the present study, ciPSCs were differentiated into cardiomyocyte by using 5-azacytidine. It was observed that the EBs when treated with differentiation media, the morphology of cells changed and spreading of EBs was extensive at the periphery (Fig. 2).

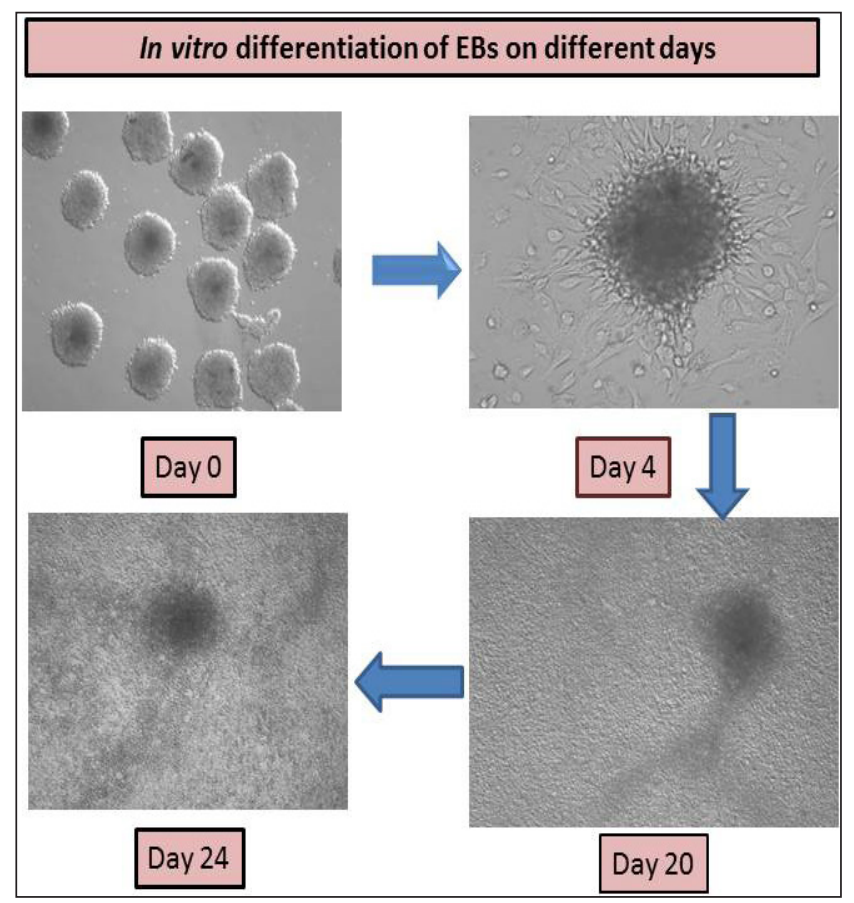

Fig. 2: In-vitro differentiation of ciPSCs on different days of culture

Cardiac specific marker expression studies by RT-PCR and gel electrophoresis revealed that MYL 2 expression started from day 8 , and continued till day 24 of differentiation (Fig. 3). It was observed that initially the expression was low but over the time of differentiation, the expression was enhanced with the highest expression on day 16 of 
differentiation and the differentiated cells were found to be immunopositive for connexin $43(\mathrm{C} \times 43)$ (Fig. 4). However, no beating clusters were observed during the entire period of differentiation.

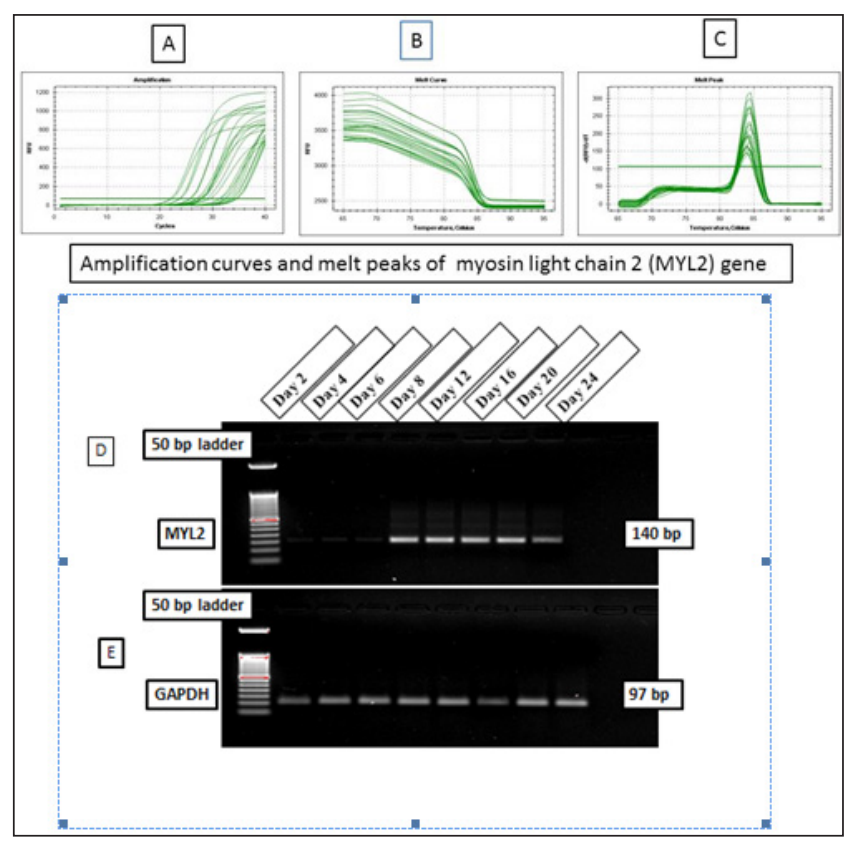

Fig. 3: Molecular characterization of MYL2 gene in differentiated embryoid bodies made from ciPSC colonies. Amplification curves (A), melt curves, (B) and melt peaks (C) of cardiac marker gene. $2 \%$ agarose gel electrophoresis of PCR amplified products of cardiac specific marker genes. (D) Cardiomyocytes specific gene MYL 2 and (E) GAPDH as a housekeeping gene

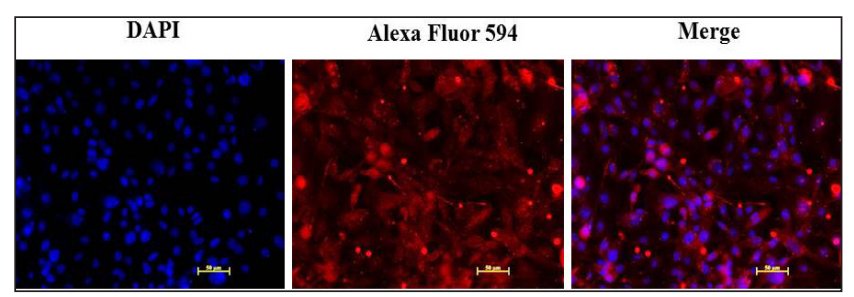

Fig. 4: immunofluorescence of cardiac gap junction protein connexin 43 (Red), DAPI nuclear staining (blue) and merged images of canine iPSC differentiated cells after 24 days of cardiac differentiation. (Scale bar $20 \mu \mathrm{m}$ )

The present study was conducted with the objective to describe the in vitro differentiation of ciPSC from canine adipose tissue derived mesenchymal stem cells. In addition, the expression of cardiac marker MYL 2 was also evaluated at various stages of differentiation.
Cardiovascular diseases now-a-days has an alarming consequences for the humans as well as animals. Mammalian heart has limited regenerative capability. During the end-stage of heart diseases, the structural damage to the cardiomyocytes incurs an irreversible functional deficit in cardiac tissue with increased burden on the surviving cardiomyocytes to meet the needs of the body, leading to several clinical manifestations. Some of the fresh developments in drug, interventional and surgical treatments do not fundamentally solve this pertinent issue. Donor availability and immunological rejection limits heart transplantation. This has shifted the focus over some of the clinical treatment of cardiovascular disease, with a great need to find more effective treatment alternatives. Stem cell compensatory replacement therapy presents a fascinating research area with a potential for treatment of cardiovascular disease (Stadtfeld et al., 2010; Vaskova et al., 2013). Although ESCs have been in consideration for longer time but, they are limited with respect to social ethics and transplantation immune rejection issues (Blau et al., 2001).

In lieu of the above mentioned drawbacks, iPSCs have attracted much attention as a novel therapeutic cell types in the field of stem cells. The iPSC have become a favored cell source to use in stem cell replacement therapies as they surpass the ethical and immune rejection problems associated with use of ESCs. Now-a-days, efforts are being invested to develop better protocols to induce iPS cells to differentiate into cardiomyocytes with cost-effectiveness, high efficiency, high purity and viability, and with a high yield to answer the needs of their clinical application. As iPSC are produced by somatic cell reprogramming they have some characteristics of primitive cells and their natural differentiation ability is relatively weaker in comparision to ESCs (Yoshida et al., 2011). For iPSC differentiation into myocardial cells in vitro, EBs formation has been considered as an important annulus with vast application (Wu et al., 2013). Although iPS cells can form a large number of EBs using the suspension method (Takahashi et al., 2003) the resulting EBs vary in size and is expensive. Instead, the hanging drop method of EB generation is cheaper and can provide better control over the size of EBs, with consistent results to help with analysis of experimental results. Researchers have been trying to find a way to enhance the efficiency of induction, including the use of chemical inducers, myocardial cell 
culture, and transfer of specific genes etc (Takahashi et al., 2003). Chemical inducers are a common choice, as these small molecules increases cell permeability, nonimmunogenic, cost-effective, easy to synthesize, preserve, and to standardize. Moreover, their effects on inhibiting and activating the function of specific proteins are often reversible and permit fine tuning by varying concentration (Cloos et al., 2008).

There were many reports suggesting that 5-azacytidine, a demethylating agent, induces differentiation of mesenchymal stem cells into cardiomyocytes in vitro (Fukuda, 2003). 5'- azacytidine can cause extensive demethylation of 5-methylcytosine and reduce DNA methyltransferase activity in the cell (Haaf and Schmid, 2000). Study on murine iPSC differentiation by media containing beta-mercaptoethanol have shown the expression of marker genes for cardiac mesoderm, and cardiomyocytes including Mesp1, GATA2 (FOG-2), GATA4, Nkx2.5, Tbx5, Tbx20, atrial natriuretic factor (ANF), MLC2a, alpha-MHC, and cardiac troponin T (Singh et al., 2019). Immunocytological assessment confirmed the expression of cardiomyocyte-typical proteins such as sarcomeric alpha-actinin, titin, cardiac troponin T, MLC2v (Mauritz et al., 2008). Many research groups have generated functional cardiomyocytes (CMs) from murine and human iPSCs in-vitro (Medvedev, 2010). Cardiomyocytes produced from iPSC are very similar in characteristics (morphology, marker expression, electrophysiological features, and sensitivity to chemicals) to the CMs of cardiac muscle and to CMs produced from differentiated ESCs. Moreover, research has shown that murine iPSCs, when injected, can repair muscle and endothelial cardiac tissues damaged by cardiac infarction (Medvedev, 2010). With regard to canine iPSCs, Joseph Wu's group has treated immunodeficient mouse models of myocardial infarction and hind limb ischemia with transplanted endothelial cells derived from ciPSCs and positively demonstrated the preclinical potential of ciPSCs (Lee et al., 2011).

The present study also supports that 5-azacytidine improves the efficiency of stem cell differentiation into cardiomyocytes by maintaining iPS cell activity, and slowing down the cell aging process. However, further studies focusing on elucidating the mechanisms involved in the effect of 5-azacytidine are of future interest.

\section{CONCLUSION}

In conclusion, the current study demonstrates that 5-azacytidine (5-aza) has markedly influenced the cardiac differentiation of canine iPSC. These findings add up to the efficient differentiation protocols of cardiac myocytes from canine iPS cell culture and their potential for therapeutic applications. The current findings suggests that treatment of ciPSC with 5-azacytidine facilitate the large-scale differentiation of cardiac myocytes which in turn will open a door for further study of heart diseases to personalized, predictive, preemptive, and precision medicine.

\section{REFERENCES}

Batalov, I. and Feinberg, A.W. 2015. Differentiation of cardiomyocytes from human pluripotent stem cells using monolayer culture: supplementary issue: stem cell biology. Biomarker insights, 10, pp.BMI-S20050.

Beltrami, A.P., Barlucchi, L., Torella, D., Baker, M., Limana, F., Chimenti, S., Kasahara, H., Rota, M., Musso, E., Urbanek, K. and Leri, A., 2003. Adult cardiac stem cells are multipotent and support myocardial regeneration. Cell, 114(6): 763-776.

Blau, H.M., Brazelton, T.R. and Weimann, J.M. 2001. The evolving concept of a stem cell: entity or function?. Cell, 105(7): 829-841.

Cloos, P.A., Christensen, J., Agger, K. and Helin, K. 2008. Erasing the methyl mark: histone demethylases at the center of cellular differentiation and disease. Genes Dev., 22(9): 1115-1140.

Davis, B.H., Morimoto, Y., Sample, C., Olbrich, K., Leddy, H.A., Guilak, F. and Taylor, D.A. 2012. Effects of myocardial infarction on the distribution and transport of nutrients and oxygen in porcine myocardium. J. Biomech. Eng., 134(10): 101-105.

Franco, D., Lamers, W.H. and Moorman, A.F. 1998. Patterns of expression in the developing myocardium: towards a morphologically integrated transcriptional model. Cardiovasc. Res., 38(1): 25-53.

Franco, D., Markman, M.M., Wagenaar, G.T., Ya, J., Lamers, W.H. and Moorman, A.F. 1999. Myosin light chain 2a and $2 \mathrm{v}$ identifies the embryonic outflow tract myocardium in the developing rodent heart. Anat. Rec. 254: 135-146.

Fukuda, K. 2003. Regeneration of cardiomyocytes from bone marrow: use of mesenchymal stem cell for cardiovascular tissue engineering. Cytotechnology, 41(2-3): 165-175.

Fukuda, K. 2003. Use of adult marrow mesenchymal stem cells for regeneration of cardiomyocytes. Bone Marrow Transplant 32: S25-S27. 
Haaf, T. and Schmid, M. 2000. Experimental condensation inhibition in constitutive and facultative heterochromatin of mammalian chromosomes. Cytogenet. Genome Res., 91(14): $113-123$.

Haaf, T. and Schmid, M. 2000. Experimental condensation inhibition in constitutive and facultative heterochromatin of mammalian chromosomes. cytogenetic and genome research., 91: 113-123.

Hailstones, D., Barton, P., Chan-Thomas, P., Sasse, S., Sutherland, C., Hardeman, E. and Gunning, P. 1992. Differential regulation of the atrial isoforms of the myosin light chains during striated muscle development. J. Biol. Chem., 267(32): 23295-23300.

Karakikes, I., Ameen, M., Termglinchan, V. and Wu, J.C. 2015. Human induced pluripotent stem cell-derived cardiomyocytes: insights into molecular, cellular, and functional phenotypes. Circ. Res., 117(1): 80-88.

Knollmann, B.C. 2013. Induced pluripotent stem cell-derived cardiomyocytes: Boutique science or valuable arrhythmia model?. Circ. Res., 112(6): 969-976.

Kubalak, S.W., Miller-Hance, W.C., O’Brien, T.X., Dyson, E. and Chien, K.R., 1994. Chamber specification of atrial myosin light chain-2 expression precedes septation during murine cardiogenesis. J. Biol. Chem., 269: 16961-16970.

Laflamme, M.A. and Murry, C.E., 2011. Heart regeneration. Nature, 473(7347): 326-335.

Lee, A.S., Xu, D., Plews, J.R., Nguyen, P.K., Nag, D., Lyons, J.K., Han, L., Hu, S., Lan, F., Liu, J. and Huang, M. 2011. Preclinical derivation and imaging of autologously transplanted canine induced pluripotent stem cells. J. Biol. Chem., 286 (37): 32697-32704.

Lundy, S. D., Zhu, W. Z., Regnier, M. and Laflamme, M.A. 2013. Structural and functional maturation of cardiomyocytes derived from human pluripotent stem cells. Stem Cells Dev., 22(14): 1991-2002.

Lyons, G.E. In situ analysis of the cardiac muscle gene program during embryogenesis. 1994. Trends Cardiovasc Med., 4: $70-77$.

Mauritz, C., Schwanke, K., Reppel, M., Neef, S., Katsirntaki, K., Maier, L.S., Nguemo, F., Menke, S., Haustein, M., Hescheler, J. and Hasenfuss, G. 2008. Generation of functional murine cardiac myocytes from induced pluripotent stem cells. Circulation, 118(5): 507.

Medvedev, S.P., Shevchenko, A.I. and Zakian, S.M. 2010. Induced pluripotent stem cells: problems and advantages when applying them in regenerative medicine. Acta Naturae, 2(2): 18-28
Moran, A.E., Forouzanfar, M.H., Roth, G.A., Mensah, G.A., Ezzati, M., Murray, C.J. and Naghavi, M. 2014. Temporal trends in ischemic heart disease mortality in 21 world regions, 1980 to 2010: the Global Burden of Disease 2010 study. Circulation, 129(14): 1483-1492.

Mummery, C.L., Zhang, J., Ng, E.S., Elliott, D.A., Elefanty, A.G., and Kamp, T.J. 2012. Differentiation of human embryonic stem cells and induced pluripotent stem cells to cardiomyocytes: a methods overview. Circ. Res., 111(3): 344-358.

Murry, C.E., Reinecke, H. and Pabon, L.M. 2006. Regeneration gaps: observations on stem cells and cardiac repair. J. Am. Coll. Cardiol., 47(9): 1777-1785.

O’Brien, T.X., Lee, K.J. and Chien, K.R. 1993. Positional specification of ventricular myosin light chain 2 expression in the primitive murine heart tube. Proc. Natl. Acad. Sci., 90: $5157-5161$.

Shimada, H., Nakada, A., Hashimoto, Y., Shigeno, K., Shionoya, Y. and Nakamura, T., 2010. Generation of canine induced pluripotent stem cells by retroviral transduction and chemical inhibitors. Mol. Reprod. Dev., 77(1): 2-2.

Singh, P., Mondal, T., Kumar, K., Das, K., Mahalakshmi. N., Madhusoodan. A.P. and Bag, S. 2019. Expression of Cardiac Specific Cell Marker in Ex Vivo Differentiated Canine iPSC. Indian J. Anim. Res., B-3829:1-5.

Stadtfeld, M., Apostolou, E., Akutsu, H., Fukuda, A., Follett, P., Natesan, S. and Hochedlinger, K. 2010. Aberrant silencing of imprinted genes on chromosome $12 \mathrm{qF} 1$ in mouse induced pluripotent stem cells. Nature, 465(7295): 175.

Takahashi, K., Tanabe, K., Ohnuki, M., Narita, M., Ichisaka, T., Tomoda, K., and Yamanaka, S. 2007. Induction of pluripotent stem cells from adult human fibroblasts by defined factors. Cell. 131(5): 861-872.

Takahashi, T., Lord, B., Schulze, P.C., Fryer, R.M., Sarang, S.S., Gullans, S.R., and Lee, R.T. 2003. Ascorbic acid enhances differentiation of embryonic stem cells into cardiac myocytes. Circulation, 107(14): 1912-1916.

Tsuji-Takayama, K., Inoue, T., Ijiri, Y., Otani, T., Motoda, R., Nakamura, S. and Orita, K. 2004 Demethylating agent, 5azacytidine, reverses differentiation of embryonic stem cells. Biochem. Biophys. Res. Commun., 323 :86-90.

Vaskova, E.A., Stekleneva, A.E., Medvedev, S.P., and Zakian, S.M. 2013. "Epigenetic memory" phenomenon in induced pluripotent stem cells. Acta Naturae, 5(4): 19-24.

Wess, G., Domenech, O., Dukes-McEwan, J., Häggström, J. and Gordon, S. 2017. European Society of Veterinary Cardiology screening guidelines for dilated cardiomyopathy in Doberman Pinschers. J. Vet. Cardiol., 19(5): 405-415. 
Wu, Y.J., Chen, S.Y., Chang, S.J. and Kuo, S.M. 2013. Enhanced differentiation of rat MSCs into cardiomyocytes with 5-azacytidine/collagen I nano-molecules. In: Proceedings of $35^{\text {th }}$ Annual International Conference of the IEEE Engineering in Medicine and Biology Society (EMBC), pp 322-325.
Yoshida, Y. and Yamanaka, S. 2011. iPS cells: a source of cardiac regeneration. J. Mol. Cell. Cardiol., 50(2): 327-332. 
